\title{
Probing Chemical Dynamics with Negative Ions
}

\author{
Daniel M. Neumark \\ Department of Chemistry, University of California, Berkeley, CA 94720, and Chemical \\ Sciences Division, Lawrence Berkeley National Laboratory, Berkeley, CA 94720

\begin{abstract}
Experiments are reviewed in which key problems in chemical dynamics are probed by experiments based on photodetachment and/or photoexcitation of negative ions.

Examples include transition state spectroscopy of bimolecular reactions, spectroscopy of open-shell van der Waals complexes, photodissociation of free radicals, and timedescribed along with representative systems that have been studied.
\end{abstract} \\ resolved dynamics in clusters. The experimental methods used in these investigations are
}

\footnotetext{
•Email: dneumark@berkeley.edu
} 


\section{Introduction}

This paper reviews work in my laboratories and elsewhere in which key problems in chemical dynamics are addressed by experiments on negative ions. Much of the research described herein was motivated by experimental and conceptual issues that arose during my doctoral work with Yuan T. Lee. As a graduate student in Yuan's research group from 1978-84, I learned that no detail could be overlooked in the design and operation of a state-of-the-art experiment. A successful scientist should not only understand how to apply to most sophisticated laser techniques to the pressing problems of the day, but also how to change mechanical pump oil in an elegant white shirt without spotting it (as Yuan delighted in demonstrating on multiple occasions). On a more conceptual level, I was inculcated with the idea that the key to understanding chemistry was to characterize the potential energy surfaces on which chemical interactions occur, and that elastic, inelastic, and reactive scattering experiments were the best way to do this, complemented by photodissociation and the occasional spectroscopy experiment. These conceptual notions fueled much of my subsequent research career, but were modified by interactions with other scientists, most notably Richard Saykally, who opened my eyes to the fact that spectroscopy provided an appealing alternative to probing potential energy surfaces, and my post-doctoral advisor, Carl Lineberger, who stimulated me to think about how seemingly obscure species, namely gas phase negative ions, could be coupled to mainstream chemical reaction dynamics and yield a new perspective on many of the questions that were central to Yuan Lee's research program.

For example, in Yuan's group, reactive scattering experiments were used to characterize the potential energy surface for a wide variety of systems ranging from $D+$ 
$\mathrm{H}_{2}$ to the reactions of $\mathrm{O}$ atoms with complex hydrocarbons. ${ }^{1-4}$ From these experiments, one hopes to determine important properties of the potential energy surface such as the heights of various barriers along the reaction coordinate and the geometries corresponding to these barriers. However, it is often problematic to extract this type of detailed information from scattering experiments, owing in part to the averaging over impact parameter intrinsic to such experiments. These considerations have motivated efforts in several laboratories ${ }^{5.7}$ to develop "transition state spectroscopy" experiments that directly probe transition states in bimolecular chemical reactions. In one of the more successful experiments of this type, ${ }^{8}$ stable negative ions similar in geometry to the neutral transition state were photodetached, and the resulting photoelectron spectra yielded resolved vibrational structure characteristic of the neutral transition state.

In another classic set of experiments, Yuan's research group carried out elastic scattering experiments involving either two rare gas atoms ${ }^{9,10}$ or a rare gas and halogen atom. ${ }^{11}$ The differential cross sections obtained in these measurements yielded some of the most accurate potential energy functions describing the relatively weak interactions between the scattering partners. Only a single surface is needed for two rare gas atoms, but three surfaces result from the interaction between a rare gas and halogen atom. While it is possible to characterize these surfaces from scattering measurements, high resolution photodetachment experiments starting from rare gas halide anions yield vibrational progressions in the low frequency anion and neutral vibrational levels for all three surfaces, ${ }^{12}$ thus providing a direct probe that is highly complementary to the scattering work. 
The bimolecular scattering experiments in Yuan's group were complemented by studies of molecular beam photodissociation, via both infrared multiphoton dissociation ${ }^{13}$ and single photon dissociation using ultraviolet lasers. ${ }^{14,15}$ These techniques were developed to a fine art and used to map out excited electronic states and their interactions with one another. Nearly all of these experiments, however, were performed on stable, closed-shell molecules. The extension of these measurements to reactive free radicals would seem to be a logical next step through the development of efficient and clean sources of these species. In practice photodissociation on molecular beams of free radicals has proved quite challenging, although some successful experiments were performed in Yuan's group ${ }^{16,17}$ and elsewhere ${ }^{18-20}$ using photolytic and pyrolytic radical sources. Again, experiments based on negative ions offer an attractive solution; one can generate a clean beam of neutral radicals by photodetachment of appropriate massselected negative ions and then photodissociate the radicals. ${ }^{21}$

While the above examples focus on using negative ions as precursors to exotic neutral species such as transition states, weakly-bound open shell clusters, and free radicals, the dynamics and spectroscopy of negative ions themselves are of considerable interest, as evidenced by the considerable recent body of work in various laboratories on water cluster anions. The gas phase femtochemistry methodology developed by Ahmed Zewail $^{22}$ showed that time-resolved measurements provide another powerful window into chemical dynamics complementary to scattering experiments, by enabling one to follow in an intuitive way the motions of nuclei in unimolecular and bimolecular reactions. These ideas motivated our research group to develop time-resolved photoelectron 
spectroscopy ${ }^{23,24}$ as a powerful means of following real-time dynamics in negative ions and negative ion clusters.

This paper is not a comprehensive review of the four types of negative ion experiments discussed above, but instead briefly describes the principles of the underlying instrumentation and offers examples of the types of systems one can study with them.

\section{Transition state spectroscopy (TSS) via negative ion photodetachment}

In negative ion TSS experiments, one measures the photoelectron (PE) spectrum of a stable negative ion similar in structure a neutral transition state. In anion PE spectroscopy, ${ }^{25}$ one photodetaches mass-selected negative ions with a fixed-frequency laser and measures the resulting kinetic energy (and sometimes angular) distribution of the ejected photoelectrons. In our group, we have generally used negative ion sources based on pulsed molecular beams and coupled to time-of-flight mass spectrometry. This pulsed ion beam instrumentation is based on work by Johnson and Lineberger, ${ }^{26}$ with various configurations implemented in our group over the years. ${ }^{27,28}$

The energy resolution of anion PE spectroscopy can be as good as 5-10 meV, generally achieved by energy-analyzing the photoelectrons with either a hemispherical analyzer or field-free time-of-flight. Somewhat poorer resolution but much higher collection efficiency is achieved using "magnetic bottle" analyzers. ${ }^{29-31}$ The latest advance, photoelectron imaging, which builds on the photofragment imaging methodology developed by Chandler ${ }^{32}$ and Parker, ${ }^{33}$ offers a versatile combination of good energy resolution, high collection efficiency, and the ability to measure photoelectron kinetic energy and angular distributions simultaneously. As such it has 
been adopted as the method of choice by several negative ion research groups. ${ }^{34-37}$

Anion PE spectroscopy has been shown to be an extremely versatile technique, since it maps out the electronic and vibrational structure of any neutral species that can be accessed by photodetachment of a negative ion. Its most frequent application has been the characterization of the ground and low-lying electronic states of free radicals, ${ }^{38,39}$ but there are also many examples of its application to metal and semiconductor cluster anions, ${ }^{40-46}$ with the primary goal of mapping out how the electronic states of the neutral clusters created by photodetachment evolve as a function of size.

In TSS experiments, photodetachment of a negative ion creates an unstable neutral species in the vicinity of a bimolecular or unimolecular transition state. ${ }^{8}$ The resulting PE spectrum can reveal resolved vibrational structure characteristic of the transition state that yields vibrational frequencies distinct from those or reactants or products, directly reflecting the nature of the chemical bonds that break and form during the course of a chemical reaction. This approach has been used by Lineberger $^{47-49}$ to study unimolecular isomerization in vinylidene and cyclo-octatetraene, and in our group to study a series of bimolecular hydrogen transfer reactions for both bare and solvated transition states. ${ }^{50-52}$ By comparison of the PE spectra to simulations on model potential energy surfaces, using either time-independent ${ }^{27}$ or time-dependent ${ }^{53}$ methods, one obtains a detailed picture of the transition state dynamics.

The application of negative ion photodetachment to transition state spectroscopy is exemplified by studies of the $\mathrm{F}+\mathrm{H}_{2}$ and $\mathrm{OH}+\mathrm{H}_{2}$ reactions by photoelectron spectroscopy of $\mathrm{FH}_{2}{ }^{-}$and $\mathrm{H}_{3} \mathrm{O}^{-}$, respectively. These are benchmark reactions that have been studied extensively by experiment and theory. The $\mathrm{F}+\mathrm{H}_{2}$ reaction, in particular, 
has defined the state-of-the-art in experimental reactive scattering studies, with increasing sophisticated crossed molecular beams experiments yielding progressively more detail on the dependence of the integral and differential cross sections on collision energy, the partitioning of product translational, vibrational, and rotational energy, and the possible reactivity of spin-orbit excited $\mathrm{F}$ atoms. ${ }^{54-63}$ Although some features of the differential cross section, namely state-selected forward scattering of the $\mathrm{HF}(\mathrm{v}=3)$ product, were originally attributed to dynamical resonances, ${ }^{54}$ these features could be largely reproduced by classical trajectory calculations ${ }^{64,65}$ on potential energy surfaces with bent as opposed to linear transition states. We therefore hoped to address the issue of the $\mathrm{FH}_{2}$ bend potential by photoelectron spectroscopy of $\mathrm{FH}_{2}$.

As shown in Fig. 1, this reaction is ideal from the TSS perspective, because electronic structure calculations indicate that the geometry of $\mathrm{FH}_{2}^{-}$is similar to that of the $\mathrm{FH}_{2}$ transition state; ${ }^{66,67}$ in both, the distance from the $\mathrm{F}$ atom to the $\mathrm{H}_{2}$ center-of- mass is relatively long, and the $\mathrm{H}-\mathrm{H}$ bond distance is similar to that in diatomic $\mathrm{H}_{2}$. These geometries reflect the fact that $\mathrm{FH}_{2}{ }^{-}$is essentially $\mathrm{F}^{-}$weakly bound to $\mathrm{H}_{2}\left(\mathrm{D}_{0} \cong 0.20 \mathrm{eV}\right)$, while the $\mathrm{F}+\mathrm{H}_{2}$ reaction has an early barrier. The calculations indicate $\mathrm{FH}_{2}{ }^{-}$is linear, so the existence or extent of bend progressions in the anion photoelectron spectrum would provide a direct probe of the bend potential at the neutral transition state.

The $\mathrm{FH}_{2}{ }^{-}$photoelectron spectrum ${ }^{68,69}$ shown in Fig. 2 indeed shows progressions in the two vibrational modes perpendicular to the reaction coordinate: the $\mathrm{H}-\mathrm{H}$ stretch and the $\mathrm{F}-\mathrm{H}-\mathrm{H}$ bend, which is more like an $\mathrm{H}_{2}$ internal rotor. The bend progression is quite extended, indicating that the $\mathrm{FH}_{2}$ transition state is bent rather than linear. This conclusion is supported by quantum mechanical simulations of the spectrum performed 
on a high level potential energy surface for the $\mathrm{F}+\mathrm{H}_{2}$ reaction. ${ }^{69}$ This system thus represents an example in which TSS resolved a key issue in a benchmark chemical reaction.

While the $\mathrm{F}+\mathrm{H}_{2}$ reaction is one of several three-atom benchmark reactions, along with $\mathrm{H}+\mathrm{H}_{2}, \mathrm{O}+\mathrm{H}_{2}$, and $\mathrm{Cl}+\mathrm{H}_{2}$, the $\mathrm{OH}+\mathrm{H}_{2} \rightarrow \mathrm{H}_{2} \mathrm{O}+\mathrm{H}$ reaction is unique as a prototypical four atom reaction, since the presence of three hydrogen atoms makes both electronic structure calculations of potential energy surfaces and the execution of scattering calculations on these surfaces tractable. As a consequence, high quality surfaces have been constructed and scattering calculations on these surfaces have been performed by several groups. ${ }^{70-74}$ Scattering experiments by Casavecchia ${ }^{70}$ and Davis ${ }^{75}$ have yielded product angular distributions and detailed vibrational energy distributions for the $\mathrm{H}_{2} \mathrm{O}$ product. These experiments are complemented by studies of the $\mathrm{OH} \cdot \mathrm{H}_{2}$ van der Waals complex by Lester and co-workers. ${ }^{76,77}$

TSS of the $\mathrm{OH}+\mathrm{H}_{2}$ reaction via photoelectron spectroscopy of $\mathrm{H}_{3} \mathrm{O}^{-}$is complicated by the existence ${ }^{78-81}$ of two anion structures: $\mathrm{H}^{-} \cdot \mathrm{H}_{2} \mathrm{O}$, which has good Franck-Condon overlap with the neutral $\mathrm{H}_{2} \mathrm{O}+\mathrm{H}$ product valley, and $\mathrm{OH}^{-} \cdot \mathrm{H}_{2}$, which overlaps the neutral transition state. As shown in Fig. 3, the $\mathrm{H}^{-} \cdot \mathrm{H}_{2} \mathrm{O}$ structure is calculated to lie $\sim 0.125 \mathrm{eV}$ lower in energy, ${ }^{82}$ which might appear problematic from the perspective of TSS. Nonetheless, the anion photoelectron spectrum $^{83}$ was quite revealing.

The top spectrum in Fig. 4 is dominated by photodetachment from $\mathrm{H}^{-} \cdot \mathrm{H}_{2} \mathrm{O}$, showing a resolved vibrational progression in which the peak spacing was slightly lower than the $\mathrm{H}_{2} \mathrm{O}$ antisymmetric stretch frequency. This progression was assigned to 
transitions to the $\mathrm{H}+\mathrm{H}_{2} \mathrm{O}$ exit valley, with the lowered frequency a signature of nonnegligible interaction between the separating products in the Franck-Condon region.

The $\mathrm{H}_{3} \mathrm{O}^{-}$photoelectron spectra show a strong angular dependence, with a low energy, broad feature appearing (bottom spectrum, Fig. 4) when the laser polarization angle is perpendicular to the direction of photoelectron detection. This feature becomes more intense when the ion source temperature is raised, suggesting it might be from the higher energy $\mathrm{OH}^{-} \cdot \mathrm{H}_{2}$ structure and therefore corresponding to photodetachment to the $\mathrm{OH}+\mathrm{H}_{2}$ transition state.

Simulations of the $\mathrm{H}_{3} \mathrm{O}^{-}$spectrum starting from both forms of the anion were carried out by de Beer et $\mathrm{al}^{83}$ in the original experimental paper and, subsequently, by several other research groups using improved potential energy surfaces and scattering methodology. Simulations from the $\mathrm{H}^{\cdot} \cdot \mathrm{H}_{2} \mathrm{O}$ structure ${ }^{83,84}$ on the best anion and neutral surfaces $^{85,86}$ available at the time of the experiment yielded an even lower frequency than that seen in Fig. 4A. This discrepancy indicates that the barrier on the model surface was too "late" along the reaction coordinate (i.e. the $\mathrm{OH} \cdot \mathrm{H}_{2}$ distance is too small at the saddle point), resulting in an overly large effect on the peak spacings in the Franck-Condon region. In fact, the saddle points on more recent $\mathrm{OH}+\mathrm{H}_{2}$ surfaces occur at larger $\mathrm{OH} \cdot \cdot \mathrm{H}_{2}$ distances (by $0.14 \AA$, on average ${ }^{72,87}$ ), and simulations of the $\mathrm{H}^{-} \cdot \mathrm{H}_{2} \mathrm{O}$ photoelectron spectrum on these new surfaces $^{81,82}$ are in better agreement with experiment, with nearly perfect agreement achieved in recent work by Collins and coworkers, ${ }^{82}$ as shown in Fig. 4A (black line). Hence, even though the $\mathrm{H}^{-} \cdot \mathrm{H}_{2} \mathrm{O}$ vibrational wavefunction has little or no overlap with the $\mathrm{OH}+\mathrm{H}_{2}$ saddle point, the nature of the vibrational structure in the photoelectron spectrum is quite sensitive to the location of the 
saddle point and therefore provides important information regarding the transition state of the reaction.

De Beer et al. ${ }^{83}$ constructed a two-dimensional potential energy surface for the $\mathrm{H}_{3} \mathrm{O}^{-}$anion and determined the 2-D vibrational wavefunctions and energy levels supported by this surface. While the $\mathrm{v}=0$ wavefunction was localized in the $\mathrm{H}^{-} \cdot \mathrm{H}_{2} \mathrm{O}$ well, as expected, the $\mathrm{v}=2$ wavefunction showed significant amplitude in the $\mathrm{OH}^{-} \cdot \mathrm{H}_{2}$ well, and simulations of the photoelectron spectrum from the $v=2$ wavefunction approximately reproduced the broad experimental feature. Hence, vibrational excitation of the anion results in considerably better overlap with the $\mathrm{OH}+\mathrm{H}_{2}$ entrance valley and transition state (see Fig. $3 \mathrm{~A}^{82}$ ), a result supported in more sophisticated simulations of the photoelectron spectrum. $^{81,82,84,88}$ Again, the most recent work by Collins ${ }^{82}$ (Fig. 4B, red line) shows excellent agreement with experiment.

\section{Anion ZEKE spectroscopy and its applications to rare gas/halogen complexes}

While anion PE spectroscopy is very general, its resolution is limited, as mentioned above, to $5-10 \mathrm{meV}$. The technique of anion zero electron kinetic energy (ZEKE) spectroscopy ${ }^{89}$ offers substantially higher resolution (as high as $0.1 \mathrm{meV}$ ) and can therefore probe lower frequency vibrational modes than conventional PE spectroscopy. In this experiment, anions are photodetached with a tunable laser, and only those photoelectrons produced with nearly zero kinetic energy are detected as a function of laser frequency. While this experiment was inspired by the neutral ZEKE experiments first carried out by Muller-Dethlefs, ${ }^{90,91}$ the physics of anion ZEKE spectroscopy is quite different as it involves direct detachment to the continuum as opposed to pulsed-field ionization of high Rydberg states. Anion ZEKE spectroscopy has been applied to the 
spectroscopy of transition states, ${ }^{92}$ radicals, ${ }^{93}$ and metal ${ }^{94,95}$ and semiconductor ${ }^{96-98}$ clusters, but here we focus on its application to rare gas-halogen complexes. ${ }^{12,99-102}$

The rare gas-halogen $(\operatorname{RgX})$ complexes play a key role in excimer lasers, in which lasing takes place between electronically excited, strongly bound charge transfer states and the repulsive wall of the weakly bound covalent ground states. ${ }^{103}$ Excimer emission has also provided spectroscopic information on the charge-transfer and covalent states. For example, in $\mathrm{KrBr}$ and $\mathrm{KrCl}$, emission from the $\mathrm{RgX}$ charge transfer states to the ground state (the $\mathrm{B} \rightarrow \mathrm{X}$ band) is broad and relatively unstructured, as is typical of boundfree transitions. However, emission studies of the $\mathrm{B} \rightarrow \mathrm{X}$ band in $\mathrm{XeBr}$ show extensive vibrational structure. ${ }^{104,105}$ The covalent states of rare gas-halogen neutrals have also been probed in a series of scattering experiments, including differential cross-sections measured in crossed molecular beam experiments by Lee and coworkers, ${ }^{11,106-108}$ which yielded potentials for several $\mathrm{RgX}$ complexes, and from integral cross section measurements by Aquilanti and co-workers. ${ }^{109}$

The neutral interactions are of interest also because they are simple examples of open shell-closed shell interactions. The effect of spin-orbit coupling on the rare gashalogen interaction potentials has been discussed at length by Aquilanti ${ }^{110}$ and Haberland. ${ }^{111}$ The ${ }^{2} \mathrm{P}_{3 / 2}$ and ${ }^{2} \mathrm{P}_{1 / 2}$ spin-orbit states of the halogen atom interact with the rare gas to give rise to three molecular electronic states, as schematically shown for $\mathrm{ArCl}$ in Fig. 5 The lower ${ }^{2} \mathrm{P}_{3 / 2}$ state is split by the electrostatic interaction into two components, corresponding to $\Omega=1 / 2$ (the $X 1 / 2$ state, in the notation used here) and $\Omega=3 / 2$ (the $I 3 / 2$ state), where $\Omega$ is the projection of the total electronic angular momentum along the internuclear axis. The upper ${ }^{2} \mathrm{P}_{1 / 2}$ halogen state gives rise to the $I I 1 / 2(\Omega=1 / 2)$ state in 
the complex.

Anion ZEKE spectroscopy of rare gas halides probes the van der Waals well region of the covalent states; this complements earlier studies of emission from excimer states. Our experiments also complement the scattering experiments because, whereas the scattering cross-sections contain information about the absolute values of the bond length and well depths of the complexes, the ZEKE spectra are sensitive only to the relative differences between the anion and neutral potentials. However, in the ZEKE spectra one can observe vibrationally-resolved photodetachment transitions to the various neutral electronic states, whereas in the crossed beam experiments the contributions of the $X 1 / 2$ and $I 3 / 2$ states to the experimental signal are not clearly separated and must be extracted by an appropriate data inversion procedure. Also, in the crossed beam experiments involving $\mathrm{Br}$ or I atoms, the $I I 1 / 2$ electronic state arising from the upper ${ }^{2} \mathrm{P}_{1 / 2}$ spin-orbit state of the halogen atom is generally not probed because the population of this state is negligible. In the ZEKE experiments, well-resolved spectra of the $I I 1 / 2$ states of the $\mathrm{KrBr}$ and $\mathrm{XeBr}$ systems are seen, and accurate potentials can be derived for these states for the first time.

ZEKE spectra for $\mathrm{ArCl}^{-}$are shown in Figs. $6 \mathrm{a}$ and $6 \mathrm{~b},{ }^{101}$ corresponding to transitions from the anion to the close-lying $X 1 / 2$ and $I 3 / 2$ neutral states and the wellseparated $I I 1 / 2$ state, respectively. The peaks labeled 1, 2, and 3 represent the vibrational origins of the three electronic transitions, while the lettered peaks are vibrational progressions involving the low-frequency anion and neutral stretching modes. Analysis of the spectra yields a very accurate electron affinity for $\mathrm{ArCl}, 29516.7 \pm 2 \mathrm{~cm}^{-1}$, and a set of fundamental vibrational frequencies such as $32 \mathrm{~cm}^{-1}$ for the $X 1 / 2$ state and $53 \mathrm{~cm}^{-1}$ for 
$\mathrm{ArCl}^{-}$. More globally, the full set of ZEKE spectra is fit to parameterized potential energy curves for the anion and three neutral states. To do this, we fix the well depth $\varepsilon$ and equilibrium bond length $R_{m}$ for one of the four states; in this case we chose the values for the $13 / 2$ from Aquilanti's total cross section measurements, ${ }^{109}$ since these agreed very well with high level calculations by Burcl et al. ${ }^{112}$ The best-fit potentials are shown in Fig. 5, and simulations of the ZEKE spectra using these potentials are shown as dotted lines in Fig. 6.

Experiments such as those described for $\mathrm{ArCl}^{-}$have yielded a set of anion and neutral potential energy curves for all binary complexes of $\mathrm{Ar}, \mathrm{Kr}$, and $\mathrm{Xe}$ with $\mathrm{Cl}, \mathrm{Br}$, and I. ${ }^{12,99,100,102}$ These diatomic potential energy curves were then used as the basis for evaluating many-body effects in clusters with multiple rare gas atoms with halogen atoms and halide anions. In particular, through the determination of electron affinities and splittings between the $X$ and $I$ states for $\operatorname{Rg}_{n} X$ clusters through a combination of ZEKE and "partially-discriminated threshold photodetachment" studies, we were able to evaluate the contributions from many-body and non-additive potential energy terms in the anion and neutral species. ${ }^{113-115}$ These terms include non-additive induction, chargeexchange quadrupole interactions, ${ }^{116}$ and charge-dispersion multipole interactions ${ }^{117}$ in the anion, as well as Axilrod-Teller triple-dipole interactions ${ }^{118}$ in the anion and neutral. These studies provide important benchmark information for understanding the role of non-additive effects in more complex systems such as water clusters. ${ }^{119}$

Recently, we have developed a new experimental arrangement to replace anion ZEKE spectroscopy based on imaging slow photoelectrons produced by anion photodetachment. This method, slow photoelectron velocity-map imaging (SEVI), offers 
comparable resolution to anion ZEKE spectroscopy but offers considerably higher data acquisition rates. ${ }^{120}$ Thus far, we have used this technique to investigate the $\mathrm{Cl} \cdot \mathrm{H}_{2}$ prereactive van der Waals complex formed by photodetachment of $\mathrm{ClH}_{2}{ }^{-}$, in which we observe hindered rotor structure that could not be resolved in the PE spectrum of the anion, ${ }^{121}$ and the methoxy radical, ${ }^{122}$ in which vibronic structure from Jahn-Teller and spin-orbit coupling is resolved that again could not be seen in the methoxide PE spectrum. $^{123,124}$

\section{Free radical photodissociation}

The vast majority of photodissociation experiments ${ }^{21}$ have been performed on stable, closed shell species or relatively unreactive radicals such as $\mathrm{NO}_{2}$. Free radicals are an extremely attractive target for photodissociation experiments, which can directly determine bond dissociation energies, map out dissociative electronic states, and probe primary product photochemistry, all of which are important in understanding the roles that radicals play in many complex chemical reaction schemes. While it might seem straightforward to measure the photodissociation dynamics of highly reactive radicals, these experiments require a "clean" source of the radical of interest, so that one can be certain that the detected photofragments come from the radical and not from the radical precursor or other unwanted species. Moreover, the radicals need to be as cold as possible to minimize the effects of internal energy on their photodissociation dynamics.

One approach to this problem is to photolyze or pyrolyze molecular beams of stable precursors to generate radicals of interest. Radical generation by photolysis has been used very effectively by Smalley, ${ }^{125}$ Miller $^{126}$ and many other groups in studies of bound-bound electronic and vibrational spectroscopy of radicals, while Chen ${ }^{127}$ has 
pioneered the "flash photolysis" technique to produce radicals from free jets. Chen and, more recently, Merkt, ${ }^{128}$ have shown that pyrolytic and photolytic radical sources coupled to free jet expansions can produce sufficiently intense and pure beams of radicals to obtain very high quality photoelectron and/or zero electron kinetic spectra. Several radical photodissociation experiments have been performed using sources of this type, 17.129-132 but the experiments and their interpretation can be complicated by the reasons given above. Recent success with radical sources based on electrical discharges coupled to pulsed slit jets have been reported by Nesbitt ${ }^{133}$ and $\mathrm{Curl}^{134}$ in high resolution infrared spectroscopy experiments; no photodissociation experiments from such sources have been reported as of yet.

We have developed a rather different approach to the problem of producing a clean source of radicals for photodissociation experiments, in which radicals are generated through laser photodetachment of a fast beam of mass-selected negative ions and then photodissociated. ${ }^{135,136}$ The overall scheme is:

$$
A B C^{-} \stackrel{h v_{1}}{\longrightarrow} A B C \stackrel{h v_{2}}{\longrightarrow} A B+C, A C+B
$$

The current configuration of the instrument ${ }^{137,138}$ is shown in Fig. 7. Anions are produced in a free jet-based source, most commonly by using a pulsed-discharge assembly attached to a pulsed piezoelectric valve. Ions are accelerated to $5-8 \mathrm{keV}$, mass selected by time-of-flight, and photodetached near the electron affinity to produce rotationally cold radicals primarily in their ground vibrational states. The resulting radicals are then photodissociated with a second laser pulse, and because of the high beam energy, the photofragments are efficiently detected with a multichannel plate detector that lies $2 \mathrm{~m}$ downstream from the photodissociation region. The detector is 
configured so that undissociated radicals are blocked, and only photofragments strike the detector. One can measure the total photofragment yield (PFY) spectrum as a function of $h v_{2}$, thereby mapping out the dissociative electronic states of the radical in question. At fixed photodissociation energies, all the photofragments can be detected in coincidence. By measuring the position and arrival time of all the fragments, one obtains the masses, kinetic energy release, and scattering angle for each photodissociation event. The current "coincidence imaging" configuration of the detector, based on the design of Zajfman and co-workers, ${ }^{139}$ uses a CCD camera to determine position information and a multi-anode photomultiplier tube to obtain crude position and precise timing information. The required timing and position information is extracted by correlating the signals from the two detectors. For photofragment channels with a large mass ratio $(>10: 1)$, it is more convenient to use a "non-coincident" detection scheme in which only the arrival times are used. $^{140}$

The power of this technique and the range of photodissociation dynamics that it can probe are exemplified by considering two seemingly similar radicals: the methoxy $\left(\mathrm{CH}_{3} \mathrm{O}\right)$ and ethoxy $\left(\mathrm{C}_{2} \mathrm{H}_{5} \mathrm{O}\right)$ radicals. These species were first observed spectroscopically in emission of ethyl nitrate photolysis in $1953 .{ }^{141}$ Both radicals are key intermediates in the combustion ${ }^{142,143}$ and atmospheric chemistry ${ }^{144}$ of hydrocarbons. From a spectroscopic perspective, the methoxy radical is one of the most widely studied polyatomic free radicals, serving as a model system for understanding the roles of spinorbit and Jahn-Teller coupling in an open shell molecule with a degenerate $\left({ }^{2} E\right)$ ground state. Numerous experiments, including electronic absorption spectra, ${ }^{145} \mathrm{PE}$ spectroscopy, ${ }^{38,123,124}$ dispersed and laser induced fluorescence (LIF), ${ }^{125,146-151}$ stimulated 
emission pumping (SEP), ${ }^{152,153}$ and vibrational spectroscopy ${ }^{154}$ have provided considerable information regarding the vibronic (and, in some cases, ro-vibronic) structure of methoxy and its deuterated counterpart. The electronic spectroscopy of the ethoxy radical has also been characterized by LIF and PE spectroscopy. ${ }^{124,155-157}$

In our fast radical beam experiments on the methoxy radical, ${ }^{158.159}$ we excited the $\tilde{A}^{2} A_{1} \leftarrow \tilde{X}^{2} E$ transition in the ultraviolet and focused on the mechanism of dissociation from the excited state. The PFY spectrum for $\mathrm{CH}_{3} \mathrm{O}$ is shown in Fig. 8. The highly structured spectrum is characteristic of a predissociating excited state. Comparison with the LIF spectra obtained by Miller and co-workers ${ }^{147}$ shows that the main progression is in the $v_{3}\left(\mathrm{C}-\mathrm{O}\right.$ stretch) mode of the $\tilde{A}$ state, and that the $3_{0}^{6}$ transition is the lowest member of this progression that dissociates.

Photofragment coincidence experiments were carried out at photon energies corresponding to several of the peaks in Fig. 8. The only mass channel seen was $\mathrm{CH}_{3}+$ $\mathrm{O}$, and the resulting photofragment translation energy $\left(\mathrm{P}\left(\mathrm{E}_{\mathrm{T}}\right)\right)$ distributions are shown in Fig. 9. Two characteristic features of these distributions are that (a) they extend to the maximum allowed translational energy, and (b) they show well-resolved vibrational progressions in the $v_{2}$ umbrella mode of the $\mathrm{CH}_{3}$ fragment. The large recoil energies observed in our data are indicative of dynamics on a purely repulsive PES, in which potential energy is converted efficiently into translational kinetic energy. Ab initio calculations by Jackels ${ }^{160,161}$ predict three excited electronic states of ${ }^{4} \mathrm{~A}_{2},{ }^{4} \mathrm{E}$, and ${ }^{2} \mathrm{~A}_{2}$ symmetry, which are repulsive along the $\mathrm{C}-\mathrm{O}$ bond and correlate asymptotically to $\mathrm{CH}_{3} \mathrm{O}\left({ }^{3} \mathrm{P}\right)$. All our results for this channel are consistent with a mechanism in which non- 
adiabatic coupling between these surfaces and the optically prepared levels of the $\tilde{A}$ state leads to predissociation of $\mathrm{CH}_{3} \mathrm{O}$.

The dissociation dynamics of the ethoxy radical are considerably more complicated, in part because there are many low-lying product channels and isomerization pathways. Fig. 10 shows the potential energy landscape for the ground electronic state of ethoxy. ${ }^{162,163}$ The lowest activation barriers are for the decomposition reactions to form $\mathrm{CH}_{3}+\mathrm{CH}_{2} \mathrm{O}$ and $\mathrm{H}+\mathrm{CH}_{3} \mathrm{CHO}$, followed by the barriers for isomerization to form $\mathrm{CH}_{2} \mathrm{CH}_{2} \mathrm{OH}$ and $\mathrm{CH}_{3} \mathrm{CHOH}$. Once isomerization to $\mathrm{CH}_{2} \mathrm{CH}_{2} \mathrm{OH}$ occurs, dissociation to $\mathrm{OH}+\mathrm{C}_{2} \mathrm{H}_{4}$ becomes possible.

In contrast to $\mathrm{CH}_{3} \mathrm{O}$, virtually no photodissociation occurs at excitation energies corresponding to the structured $\tilde{B}^{2} A^{\prime} \leftarrow \tilde{X}^{2} A^{n}$ transition previously seen by LIF, for which the vibrational origin occurs at $3.618 \mathrm{eV} .^{155,156}$ The PFY spectrum measured by Choi et al. ${ }^{164}$ showed only a higher energy, unstructured band starting around $4.7 \mathrm{eV}$. Recent electronic structure calculations in our group ${ }^{140}$ attribute this band to the $\tilde{C}^{2} A^{\prime \prime} \leftarrow \tilde{X}^{2} A^{\prime \prime}$ transition, corresponding to electronic excitation from a C-H $\sigma$-bonding orbital on the methyl group to a non-bonding $p-\pi$ orbital on the $\mathrm{O}$ atom.

Fragment mass distributions for the photodissociation within this band using both coincident and non-coincident detection schemes show three channels with approximately equal yields: $\mathrm{OH}+\mathrm{C}_{2} \mathrm{H}_{4}, \mathrm{CH}_{3}+\mathrm{CH}_{2} \mathrm{O}$, and $\mathrm{H}+\mathrm{CH}_{3} \mathrm{CHO}$; the last channel can only be detected by non-coincident methods because of the large disparity in fragment masses. $\mathrm{P}\left(\mathrm{E}_{\mathrm{T}}\right)$ distributions for the first two channels are shown in Fig. 11, ${ }^{140}$ along with the anisotropy parameter $\beta\left(E_{T}\right)$. The $P\left(E_{T}\right)$ distribution for $\mathrm{OH}+\mathrm{C}_{2} \mathrm{H}_{4}$ peak 
below $0.5 \mathrm{eV}$, whereas those for $\mathrm{CH}_{3}+\mathrm{CH}_{2} \mathrm{CO}$ and $\mathrm{H}+\mathrm{CH}_{3} \mathrm{CHO}$ (not shown) show distinct maxima around $1-1.5 \mathrm{eV}$.

The $\mathrm{P}\left(E_{\mathrm{T}}\right)$ distributions for $\mathrm{C}_{2} \mathrm{H}_{5} \mathrm{O}$ stop well short of the maximum allowed value of $E_{\mathrm{T}}$ (in the range of 4-5 eV, depending on the channel and photon energy), in contrast to the $\mathrm{P}\left(E_{\mathrm{T}}\right)$ distributions for $\mathrm{CH}_{3} \mathrm{O}$ photodissociation. Hence, it appears that ethoxy does not undergo dissociation on repulsive, excited state surfaces. In fact, the distributions in Fig. 11 look superficially like those expected for internal conversion on the ground state followed by statistical dissociation, in which vibrational energy randomization occurs prior to dissociation. For example, Fig. 10 shows that there is an exit barrier with respect to products for the $\mathrm{CH}_{3}+\mathrm{CH}_{2} \mathrm{O}$ channel, and $\mathrm{P}\left(\mathrm{E}_{\mathrm{T}}\right)$ distribution for this channel peaks well away from zero. Likewise, there is only a small exit barrier for the dissociation of $\mathrm{CH}_{2} \mathrm{CH}_{2} \mathrm{OH}$ to $\mathrm{OH}+\mathrm{C}_{2} \mathrm{H}_{4}$, consistent with the $\mathrm{P}\left(\mathrm{E}_{\mathrm{T}}\right)$ distribution peaking near zero. However, a closer look indicates that this simple picture is inadequate. In statistical dissociation, one expects the $\mathrm{P}\left(\mathrm{E}_{\mathrm{T}}\right)$ distribution to peak at a fraction of the barrier height, ${ }^{165,166}$ not a factor greater than unity as appears to be the case here for $\mathrm{CH}_{3}+$ $\mathrm{CH}_{2} \mathrm{O}$. The presence of significant $\mathrm{OH}$ elimination is even more troublesome; on the ground state, this channel requires isomerization over a high barrier with a tight transition state, so it should be strongly disfavored if the dynamics were statistical.

One can rationalize the $\mathrm{P}\left(\mathrm{E}_{\mathrm{T}}\right)$ distribution for the $\mathrm{CH}_{3}+\mathrm{CH}_{2} \mathrm{O}$ channel as resulting from dissociation so fast on the ground state surface that vibrational energy randomization is incomplete, resulting in more translational energy than would otherwise be expected. The $\mathrm{OH}$ channel is more problematic and appears to involve significant excited state dynamics. For example, the nature of the electronic transition, in which a C- 
$\mathrm{H}$ bonding electron is excited to a non-bonding orbital, may lower the isomerization barrier to $\mathrm{CH}_{2} \mathrm{CH}_{2} \mathrm{OH}$ on the upper state. Dissociation can then continue on an excited state, or proceed through a conical intersection to the $\mathrm{CH}_{2} \mathrm{CH}_{2} \mathrm{OH}$ local minimum on the ground state, after which dissociation to $\mathrm{OH}+\mathrm{C}_{2} \mathrm{H}_{4}$ occurs; dynamics of this type are often postulated in organic photochemistry. ${ }^{167}$ In any case, it is apparent that the ethoxy dissociation dynamics are considerably more complicated and, at this point, less wellcharacterized than those of the methoxy radical.

\section{Time-resolved photoelectron spectroscopy of negative ions}

The development of femtosecond time-resolved methods for the study of gasphase molecular dynamics is founded upon the seminal studies of Zewail and coworkers, ${ }^{22}$ as recognized in 1999 by the Nobel Prize in Chemistry. This methodology has been applied to chemical reactions ranging in complexity from bond-breaking in diatomic molecules to dynamics in larger organic and biological molecules, and has led to breakthroughs in our understanding of fundamental chemical processes. Photoexcited poly-atomic molecules and anions often exhibit quite complex dynamics involving the redistribution of both charge and energy. ${ }^{2 *}$ These processes are the primary steps in the photochemistry of many polyatomic systems, are important in photobiological processes such as vision and photosynthesis, "and underlie many concepts in molecular electronics."

Femtosecond time-resolved photoelectron spectroscopy (fs-TRPES) has shown itself to be an extremely versatile technique for probing time-resolved dynamics. In these experiments, one or more fs pump laser pulses creates a non-stationary wavepacket on either an excited or ground state of the species under study, the probe laser generates free electrons through photoionization or photodetachment, and the electron kinetic 
energy (and/or angular) distribution is measured as a function of time. TRPES, which has been reviewed several times in recent years, ${ }^{23,24,168-170}$ has the attractive feature of being able to follow dynamics along the entire reaction coordinate for rather complex dynamical mechanisms.

TRPES was first applied to neutral species, ${ }^{171-174}$ but in our group, we have focused on applications of TRPES to negative ions, with particular emphasis on dynamics in negative ion clusters. While the more complex sources and lower number densities in negative ion experiments present challenges that are absent in the neutral experiments, detachment energies are generally significantly lower than ionization energies in neutral species and are typically overcome with easily generated probe laser wavelengths. For example, in studies of radiationless transitions, most neutral TRPES experiments "lose track" of dynamics once internal conversion to the ground state occurs, this limitation does not hinder negative ion experiments. In addition, studies of clusters are straightforward in negative ion experiments because the ions can be mass-selected prior to their interaction with the laser pulses; analogous neutral studies require collecting photoions in coincidence with photoelectrons so that the identity of the ionized species can be ascertained. Hence, for example, several groups have used TRPES to measure electronic relaxation dynamics in size-selected metal clusters. ${ }^{175-177}$

Clusters of $\mathrm{I}_{2}^{-}$with various solvent species have proved to be extremely attractive model systems from the perspective of time-resolved dynamics in negative ions. In seminal experiments by Lineberger and co-workers, ${ }^{178-182}$ the $\mathrm{I}_{2}^{-}$chromophore in $\mathrm{I}_{2}^{-}\left(\mathrm{CO}_{2}\right)_{\mathrm{n}}$ or $\mathrm{I}_{2}^{-}(\mathrm{Ar})_{\mathrm{n}}$ was excited from its ground $X^{2} \Sigma_{u, 1 / 2}^{+}$state to the repulsive $A^{\prime 2} \Pi_{g .1 / 2}$ state. While the quantum yield for fragmentation to $\mathrm{I}^{+} \mathrm{I}^{-}$is unity for bare $\mathrm{I}_{2}^{-}$, 
in clusters the recoiling photofragments can lose energy by collisions with the solvating species, followed by recombination and vibrational relaxation. By measuring the ionic photofragment mass distribution in one-photon and time-resolved pump-probe experiments, the effect of the number and type of solvent species on the caging and recombination dynamics was probed in considerable detail. These experiments can be compared directly to time-resolved experiments on $\mathrm{I}_{2}{ }^{-}$in solution carried out by Barbara and co-workers. ${ }^{183}$

In our group, we have performed femtosecond TRPES experiments on $\mathrm{I}_{2}^{-}\left(\mathrm{CO}_{2}\right)_{\mathrm{n}}$ and $\mathrm{I}_{2} \cdot(\mathrm{Ar})_{\mathrm{n}}$ clusters using pump and probe laser pulses in order to gain further insights into these dynamics. ${ }^{184-187}$ However, the interpretation of these experiments was complicated by the myriad of concurrent recombination and relaxation processes. A considerably cleaner picture of the dynamics in these clusters was obtained with a more complex, three-pulse experiment as outlined in Fig. 12.

In these experiments, a vibrationally excited wavepacket is created on the $\mathrm{I}_{2}{ }^{-}$ $X^{2} \Sigma_{u, 1 / 2}^{+}$ground state by means of femtosecond stimulated emission pumping (FSEP). ${ }^{188}$ Here, a sequence femtosecond pump and dump pulses excites the $\mathrm{I}_{2}^{-}$from the $X$ state to the repulsive $A^{\prime 2} \Pi_{g .1 / 2}$ state and then drives some of the dissociating wavepacket back down to the $X$ state, creating a wavepacket on the ground state with average excitation energy $E_{e x c}=h v_{p u m p}-h v_{d u m p} . E_{e x c}$ can be varied by tuning $h v_{d u m p}$. The resulting wavepacket dynamics are monitored by photodetachment with a third laser pulse, $h v_{\text {probe }}$, and measurement of the resulting photoelectron spectrum. The measured photoelectron kinetic energy is sensitive to the phase of the wavepacket and, as can be seen from Fig. 12 , electrons with highest $\mathrm{eKE}$ are produced when the wavepacket is at its inner turning 
point. By monitoring these highest eKE electrons, one observes oscillations at short times representing coherent motion of the wavepacket.

For bare $\mathrm{I}_{2}^{-}$, in which no vibrational relaxation occurs, one simply observes dephasing and rephasing dynamics characteristic of a diatomic molecule vibrating in an anharmonic potential, with the oscillation frequency reflection the energy difference between adjacent vibrational levels comprising the wavepacket. ${ }^{188}$ Hence, the oscillation frequency decreases significantly as $E_{\text {exc }}$ is increased. In clusters of $\mathrm{I}_{2}{ }^{-}$with $\mathrm{Ar}$ or $\mathrm{CO}_{2}$, one still observes oscillations associated with coherent wavepacket motion, although these dephase irreversibly, with time constants of 3-4 ps in $\mathrm{I}_{2}^{-}\left(\mathrm{CO}_{2}\right)_{4,5}$ clusters and 8-10 ps in $\mathrm{I}_{2}^{-}(\mathrm{Ar})_{\mathrm{n} \leq 12}$ clusters, ${ }^{189-191}$ as shown in Fig. 13. More significantly, the oscillation frequency increased with time, reflecting vibrational relaxation of the $I_{2}{ }^{-}$in an anharmonic potential, where the level spacing increases as the vibrational quantum number drops. The increase in frequency is as large as $20 \mathrm{~cm}^{-1}$ for $\mathrm{I}_{2}^{-}\left(\mathrm{CO}_{2}\right)_{4}$ at $E_{\text {exc }}=0.57$ $\mathrm{eV}$; this implies that the wave packet loses at least $0.35 \mathrm{eV}$ of vibrational energy in $\sim 3.5$ ps while maintaining its coherence. On a longer time-scale, one can follow the extent of cluster fragmentation subsequent to FSEP excitation, ${ }^{192}$ thereby extracting dissociation energies for each solvent molecule.

More recently, we have used photoelectron imaging to investigate the spectroscopy and time-resolved dynamics of $\left(\mathrm{H}_{2} \mathrm{O}\right)_{n}{ }^{-}$clusters and their deuterated analogs. ${ }^{193-195}$ This work is motivated by the extensive literature on the bulk hydrated electron, $e_{a q}{ }^{196}$ This species has been recognized as an important participant in radiation chemistry and as an important reagent in charge-induced reactivity, molecular-biological processes, and condensed phase chemistry. The significance of $e_{a q}^{-}$has sparked much 
experimental activity aimed at characterizing its energetics and dynamics in solution through various spectroscopic methods. Additionally, it stands as a most fundamental quantum-mechanical solute, and modeling its properties through various theoretical approaches provides new insights into condensed-phase dynamics. The hydrated electron exhibits a broad absorption band peaking at $720 \mathrm{~nm}^{197}$ that is generally attributed to excitation from an occupied " $s$ " state to a broadened manifold of " $p$ " states within a nonspherical cavity of water molecules. ${ }^{198,199}$ Time-resolved dynamics experiments show that, subsequent to electronic excitation, the transient absorption spectrum of $e_{a q}^{-}$is shifted much further to the infrared, with recovery of the equilibrium absorption occurring within 1-2 ps. ${ }^{200-204}$ However, interpretation of the dynamics underlying this spectral evolution has been controversial. Two different mechanisms have been put forth: the "adiabatic solvation" mechanism, ${ }^{205,206}$ in which the $p-s$ internal conversion (IC) lifetime is on the order of $1 \mathrm{ps,}$, and the "non-adiabatic solvation" mechanism in which the IC lifetime is $50 \mathrm{fs}^{204.207}$

Studies of water cluster anions, $\left(\mathrm{H}_{2} \mathrm{O}\right)_{n}{ }^{-}$, offer a parallel approach to understanding essential features of electron hydration by probing in detail the sizedependent photophysical properties of these clusters. These clusters were first detected in mass spectroscopy experiments by Haberland. ${ }^{208,209}$ Since then, the photoelectron (PE), ${ }^{194,210,211}$ electronic absorption, ${ }^{212,213}$ and vibrational absorption ${ }^{214,215}$ spectra of water cluster anions have been investigated in order to assess the nature of the binding of the excess electron and whether and at what size these clusters possess the characteristics of $e_{a q .}$. Interpretation of these experiments has been facilitated by many theoretical studies of these anions using quantum path integral, molecular dynamics (MD), and 
electronic structure methods. ${ }^{216-221}$ The vibrational spectroscopy experiments of Johnson and co-workers ${ }^{215,222}$ have proved particularly valuable, showing, for example, that in small clusters the excess electron binds primarily at the surface to a "double-acceptor" water molecule with two dangling $\mathrm{H}$ atoms.

Time-resolved PE spectroscopy experiments, carried out in our group ${ }^{193-195}$ and by Johnson ${ }^{213}$ and Zewail, ${ }^{223}$ have provided direct information on the excited state lifetimes in these clusters that have led to new insights into relaxation in the bulk. Fig. 14 shows the two-photon excitation scheme used in our experiments(a) and, in (b), the results for $\left(\mathrm{D}_{2} \mathrm{O}\right)_{25}$. The $p$-s transition is excited at $1.0 \mathrm{eV}$ and the upper state dynamics are probed at $3.1 \mathrm{eV}$. The resulting pump-probe signal yields photoelectrons at energy $\mathrm{D}$. In addition, the probe laser can directly photodetach ground state ions, yielding photoelectrons at the lower energy A. Hence, signal at energies D and A measures the $p$ state and $s$-state populations, respectively. In Fig. 14b, the signal at D rises according to the cross-correlation of the pump and probe pulses and falls with a time constant of $\sim 400$ fs. Feature A evolves oppositely; it is first depleted then rises with a time constant equal to the fall time of $\mathrm{D}$. No other dynamics (such as autodetachment) occur on this time scale, as evidenced by the fact that the lower energy features B and C are constant. ${ }^{195}$ Hence, our measurements provide an unambiguous value for the lifetime of the cluster $p$ state and show that it decays solely by internal conversion to the $s$-state.

Measured excited state lifetimes $\tau$ for a range of cluster sizes up to $n \sim 200$ are plotted vs. 1/ $n$ in Fig. 15. Results are shown for both isotopomers, and for isomers labeled I and II. Isomer I clusters (such as $\left(\mathrm{D}_{2} \mathrm{O}\right)_{25}{ }^{-}$in Fig. 14b), have higher vertical detachment energies than isomer II clusters, which can only be made under unusual ion 
source conditions. We have previously assigned isomer I and II clusters to states in

which the excess electron is internalized and surface-bound electrons, respectively, ${ }^{194,224}$ although this assignment has been questioned recently. ${ }^{220}$ In any case, we find a strong isotope effect for both types of isomers, $\tau\left(\mathrm{H}_{2} \mathrm{O}\right)_{n} / \tau\left(\mathrm{D}_{2} \mathrm{O}\right)_{n} \sim 2$.

The key finding in Fig. 15 is that isomer I lifetimes for $n \geq 25$ lie on a straight line and that extrapolation to the infinite size limit leads to internal conversion lifetimes of 50 and $70 \mathrm{fs}$ for $\mathrm{H}_{2} \mathrm{O}$ and $\mathrm{D}_{2} \mathrm{O}$, respectively. These values match the $\mathrm{IC}$ lifetimes for the bulk hydrated electrons extracted by Wiersma and co-workers ${ }^{204}$ within the context of the non-adiabatic solvation model of the hydrated electron. Hence, our measurements on finite clusters are in much better agreement with that picture than with the adiabatic solvation model, thereby offering strong support to the non-adiabatic solvation model. Isomer I clusters with $n \leq 25$ show shorter relaxation times than expected based on the linear fit in Fig. 15. Detailed analysis of the features B and C for these smaller clusters shows that the excited state undergoes autodetachment in addition to internal conversion, thereby providing a parallel pathway for upper state decay. Finally, lifetimes for isomer II clusters show no detectable dependence on cluster size, consistent with their assignment as surface states in which the excess electron is only weakly coupled to the solvent network. Overall, this set of experiments represents an exemplary case of elucidating an important bulk phenomenon, electron hydration, through studies of finite clusters.

\section{Outlook}

Future studies of chemical dynamics using negative ions will benefit greatly from new spectroscopic tools developed in several laboratories to characterize these species in 
more detail. For example, in photoelectron spectroscopy of negative ions, the more one knows about the geometry and vibrational frequencies of the anions, the more information one can extract from the photoelectron spectrum about the neutral species created by photodetachment, regardless of whether this species is a radical, a transition state, or a cluster. While high resolution, rotationally-resolved infrared spectra have been obtained for only a handful of small negative ions, ${ }^{225-227}$ vibrationally-resolved spectra have been obtained for a host of negative ion clusters using variants of infrared ion photodissociation spectroscopy, ${ }^{228}$ yet another field with its origins in Yuan Lee's laboratories. ${ }^{229}$ Vibrationally resolved spectra have yielded detailed pictures of how, for example, water molecules hydrate an atomic or molecular anion, ${ }^{230}$ or, as mentioned above, how excess electrons bind to small water clusters. ${ }^{215}$ Until very recently, infrared photodissociation spectroscopy of positive and negative ions was limited to relatively high frequency vibrations (typically above $2500 \mathrm{~cm}^{-1}$ ). However, infrared free electron lasers and newly developed table-top systems have now extended the accessible spectral range to as low as $600 \mathrm{~cm}^{-1}$, making it possible to probe the very low-frequency vibrations associated with the shared proton in strongly hydrogen-bonded ions. ${ }^{231-233}$ These breakthroughs in structural characterization will play a key role as increasingly complex negative ions are investigated in gas phase spectroscopy and dynamics experiments. For example, there has been considerable interest in the PE spectroscopy of negatively charged biomolecules since the pioneering work of Bowen, ${ }^{234}$ who found that intact DNA bases could support an excess electron in dipole-bound states. Subsequent photoelectron spectra of negatively-charged dimers of DNA bases by this group showed strong evidence for barrier-free proton transfer in the anion; ${ }^{235}$ there are 
clear analogies between this work and the PE spectroscopy of anionic transition state precursor species described in Section II. Very recently, Zewail ${ }^{236}$ has used timeresolved photoelectron spectroscopy to follow the dynamics of a complex, substituted phenolate anion that models the chromophore of the photoactive yellow protein. Overall, it appears that the arsenal of spectroscopic and dynamical techniques developed for very fundamental studies of negative ions and the transient species generated by photodetachment will have an ever-expanding impact in the near future.

\section{Acknowledgements}

This research has been supported by the Air Force Office of Scientific Research, the National Science Foundation, the Department of Energy, and the Office of Naval Research. The work presented here could not have been done without the efforts of many devoted graduate students, post-doctoral fellows, and collaborators, and I am indebted to all of them for their contributions over the years. 